\title{
Avaliação do grau de conhecimento dos Cirurgiões Dentistas sobre a utilização dos bisfosfonatos e seus efeitos adversos: Estudo descritivo
}

\author{
Assessment of the degree of knowledge of Dentists about the use of bisphosphonates and their \\ adverse effects: Descriptive study \\ Evaluación del grado de conocimiento de los Cirujanos Dentistas sobre el uso de bifosfonatos y sus \\ efectos adversos: Estudio descriptivo
}

Recebido: 02/05/2021 | Revisado: 10/05/2021 | Aceito: 11/05/2021 | Publicado: 23/05/2021

Sâmya Rayne Clementino de Sousa ORCID: https://orcid.org/0000-0003-2136-1211 Centro Universitário Maurício de Nassau, Brasil E-mail: samysousa@gmail.com

Juliana Campos Pinheiro

ORCID: https://orcid.org/0000-0001-5687-7635 Universidade Federal do Rio Grande do Norte, Brasil E-mail: dra.julianacpinheiro@gmail.com Joaquim Felipe Junior

ORCID: https://orcid.org/0000-0001-9653-3386 Universidade Federal do Rio Grande do Norte, Brasil E-mail: joaquim.felipe354@gmail.com

Danielle Machado Farias

ORCID: https://orcid.org/0000-0003-1829-0010 Universidade Federal do Rio Grande do Norte, Brasil E-mail: danifarias54@gmail.com

Dennys Ramon de Melo Fernandes Almeida

ORCID: https://orcid.org/0000-0002-4686-4379 Universidade Federal do Rio Grande do Norte, Brasil E-mail: dennysfernandes@ymail.com

Jabes Gennedyr da Cruz Lima ORCID: https://orcid.org/0000-0001-7420-7686 Universidade Federal do Rio Grande do Norte, Brasil E-mail: jabes.gennedyr@hotmail.com

Cristianne Kalinne Santos Medeiros ORCID: https://orcid.org/0000-0003-2439-2523 Universidade Federal do Rio Grande do Norte, Brasil E-mail: cristiannekalinne@gmail.com

Marvin Felipe Oliveira

ORCID: https://orcid.org/0000-0001-5687-7635 Faculdade IDE, Brasil E-mail: marvinfelipe156@gmail.com

Lucas Coimbra de Assis ORCID: https://orcid.org/0000-0002-2099-7149

Universidade Tiradentes, Brasil

E-mail: lucascoimbra90@gmail.com

Izabella Costa Calassa

ORCID: https://orcid.org/0000-0002-0769-6835

Centro Universitário Maurício de Nassau, Brasil

E-mail: izabellacalassa@gmail.com

Suany Lara Soares Araújo

ORCID: https://orcid.org/0000-0002-8290-8527

Centro Universitário Maurício de Nassau, Brasil E-mail: suanyaraujo2507@gmail.com

Rafaella Bastos Leite

ORCID: https://orcid.org/0000-0002-3304-120X Centro Universitário Maurício de Nassau, Brasil E-mail: rrafaella_bastos@hotmail.com Josélia Silva Leite

ORCID: https://orcid.org/0000-0001-7736-7896 Centro Universitário Maurício de Nassau, Brasil E-mail: joseliasilva@gmail.com 


\begin{abstract}
Resumo
O Cirurgião Dentista deve estar capacitado a conhecer os riscos relacionados a utilização dos bisfosfonatos. Este estudo tem como objetivo avaliar o conhecimento e condutas dos Cirurgiões Dentistas acerca da utilização dos bisfosfonatos e seus efeitos adversos. A amostra foi constituída por 60 Cirurgiões Dentista especialistas nas áreas de Cirurgia $(n=20)$, Implantodontia $(n=20)$ e Periodontia $(n=20)$, que responderam a um questionário estruturado e validado por Albuquerque (2014) com 12 perguntas, possuindo questões pessoais, tópicos sobre a anamnese realizada por estes profissionais e sobre o conhecimento sobre os bisfosfonatos, incluindo indicações, efeitos adversos e tratamentos empregados quando necessário. Dos 60 participantes, 88,3\% já atenderam pacientes que relatam o uso de Bisfosfonatos, porém apenas 33,3\% conhecem as complicações orais do seu uso. Foi concluído que houve um número considerável de Cirurgiões Dentistas cientes da existência dos bisfosfonatos, uma pequena parcela sabe sobre as complicações orais desse medicamente e sua forma de tratamento.
\end{abstract}

Palavras-chave: Bisfosfonatos; Cirurgia bucal; Osteonecrose.

\begin{abstract}
The Dental Surgeon must be able to know the risks related to the use of bisphosphonates. This study aims to evaluate the knowledge and conduct of dental surgeons about the use of bisphosphonates and their adverse effects. The sample consisted of 60 Dental Surgeons specialized in the areas of Surgery $(n=20)$, Implantology $(n=20)$ and Periodontics $(n=20)$, who answered a structured questionnaire and validated by Albuquerque (2014) with 12 questions, having personal questions, topics about the anamnesis performed by these professionals and about the knowledge about bisphosphonates, including indications, adverse effects and treatments used when necessary. Of the 60 participants, $88.3 \%$ have seen patients who report the use of Bisphosphonates, however only $33.3 \%$ know the oral complications of its use. It was concluded that there were a considerable number of Dental Surgeons aware of the existence of bisphosphonates, a small portion knows about the oral complications of this medication and its form of treatment.
\end{abstract}

Keywords: Bisphosphonates; Surgery oral; Osteonecrosis.

\title{
Resumen
}

El Cirujano Dentista debe poder conocer los riesgos relacionados con el uso de bifosfonatos. Este estudio tiene como objetivo evaluar el conocimiento y la conducta de los cirujanos dentistas sobre el uso de bifosfonatos y sus efectos adversos. La muestra estuvo conformada por 60 Cirujanos Dentistas especializados en las áreas de Cirugía $(n=20)$, Implantología $(n=20)$ y Periodoncia $(n=20)$, quienes respondieron un cuestionario estructurado y validado por Albuquerque (2014) con 12 preguntas, habiendo cuestiones personales, temas sobre la anamnesis realizada por estos profesionales y sobre el conocimiento sobre bifosfonatos, incluyendo indicaciones, efectos adversos y tratamientos utilizados cuando sea necesario. De los 60 participantes, el 88,3\% ha visto pacientes que reportan el uso de Bifosfonatos, sin embargo solo el 33,3\% conoce las complicaciones orales de su uso. Se concluyó que existía un número considerable de Cirujanos Dentistas conscientes de la existencia de bifosfonatos, una pequeña porción conoce las complicaciones orales de este medicamento y su forma de tratamiento.

Palabras clave: Bifosfonatos; Cirugía bucal; Osteonecrosis.

\section{Introdução}

Os bisfosfonatos (BFs) são medicamentos amplamente utilizados no tratamento de doenças relacionadas à perda de mineral ósseo devido ao aumento da reabsorção óssea. O uso destes medicamentos atualmente é majoritariamente voltado para o tratamento de pacientes com osteoporose, Síndrome de Paget, como também doenças ósseas benignas e câncer de mama, próstata, pulmão, tanto em administração oral quanto endovenosa (Marx, 2003; Ruggiero, 2006; Tolentino, 2018).

Mesmo com seus benefícios, os BFs possuem efeitos colaterais, sendo um dos principais efeitos adversos a osteonecrose associada aos bifosfonatos, mundialmente conhecida como BRONJ (Bisphoshponate-related osteonecrosis of the jaw - Osteonecrose maxilar associada ao uso de bifosfonatos) e posteriormente renomeada para MRONJ (Medication-related osteonecrosis of the jaw - Osteonecrose maxilar associada ao uso de medicamentos). A osteonecrose se inicia pelo osso alveolar e pode estender-se ao bordo inferior ou ramo mandibular, ao zigomático ou a parede do seio maxilar (Marx, 2003; Yoo, 2010; Hellstein, 2011; Chianesi, 2018).

Os BFs têm elevada afinidade pelo tecido ósseo, ligando-se ao cálcio nos cristais de hidroxiapatita. Tal afinidade pela hidroxiapatita explica a ação farmacológica específica desses compostos sobre os tecidos minerais. Os BFs são utilizados com o objetivo de promover a remodelação óssea, atuando na inibição da reabsorção óssea, estes processos estão associados a diminuição da expressão de proteínas como RANKL, essencial para a diferenciação dos osteoclastos e o aumento da expressão 
da osteoprotegerina (OPG), inibidor da diferenciação de osteoclastos (Greenber, 2008; Hinchy, 2013; Toksvig-Larsen, 2013; Carvalho, 2018).

Clinicamente osteonecrose é perceptível por apresentar uma exposição óssea da maxila ou da mandíbula. Esses quadros clínicos podem ser variáveis, podendo ser sintomáticos ou por vezes assintomáticos, como também em relação ao tempo de evolução que pode ser por semanas ou meses. Quando se tem um quadro sintomático, ele se apresenta com sintomatologia dolorosa localizada, mobilidade dentária, fístulas que não cicatrizam drenagem de pus e edema de tecido mole (Carvalho, 2008; Kim, 2013; Rigo, 2017).

O protocolo de tratamento para o osteonecrose dos maxilares causada pelo uso dos BFs é direcionado para cada caso dependendo do grau clínico da doença. O tratamento é integrado e envolve o uso de antibióticos, irrigação local com solução antimicrobiana, debridamento local da ferida, sequestrectomia cirúrgica, uso de plasma rico em plaquetas, como também a oxigenação hiperbárica (Rodan, 1996; Sampaio, 2010; Martins, 2009; Brozoski, 2012).

Desta forma, torna-se fundamental que os Cirurgiões Dentistas tenham o conhecimento sobre a associação dos BFs com a osteonecrose, reconhecendo o grupo de pacientes de risco, assim como as formas de tratamento que podem ser propostas aos pacientes. Por isso, este estudo tem o objetivo de verificar o nível de conhecimento dos profissionais especialistas em áreas odontológicas cirúrgicas sobre o tratamento com BFs e seus efeitos adversos.

\section{Metodologia}

Trata-se de um estudo transversal, de abordagem quantitativa e qualitativa, realizada por meio de um questionário estruturado feito por Albuquerque (2014). A Pesquisa foi aprovada pelo CEP através do parecer de número 3.916.326. O questionário coletou informações referentes ao grau de conhecimento dos Cirurgiões Dentistas relacionados aos BFs e suas implicações bucais, assim como sobre a conduta clinica a ser tomada. Os critérios de inclusão foram: Cirurgiões Dentistas atuantes nas especialidades de Cirurgia, Implantodontia e Periodontia, da cidade de João Pessoa, Paraíba. Já os critérios de exclusão foram: Cirurgiões Dentistas que preencherem o questionário da pesquisa inadequadamente e que não assinaram o termo de consentimento livre e esclarecido.

Por se tratar de dados categóricos, a descrição das características da amostra e as respostas sobre BFs foram apresentadas em tabelas de distribuição de frequência absolutas e relativas. As análises foram realizadas com auxílio do programa R.R Core Team (R: A language and environment for statistical computing. R Foundation for Statistical Computing, Vienna, Austria, 2020).

\section{Resultados e Discussão}

Na Tabela 1 e Figuras 1, 2, 3, 4 e 5 são apresentadas as características da amostra. 
Tabela 1. Perfil dos Cirurgiões Dentistas da amostra.

\begin{tabular}{|l|l|c|}
\hline Variável & Categorias & Frequência (\%) \\
\hline \multirow{2}{*}{ Idade } & Até 30 anos & $13(21,0 \%)$ \\
& De 31 a 49 anos & $2(3,3 \%)$ \\
\hline \multirow{2}{*}{ Tempo de formado } & 50 anos & $52(86,7 \%)$ \\
& Até 1 ano & $2(3,3 \%)$ \\
& De 2 a 5 anos & $5(8,3 \%)$ \\
& De 6 a 10 anos & $1(1,7 \%)$ \\
\hline \multirow{2}{*}{ Local de atuação } & Mais de 10 anos & $30(50,0 \%)$ \\
& Consultório particular & $20(33,3 \%)$ \\
& Serviço público de saúde & $10(16,7 \%)$ \\
\hline${ }^{1}$ Área da Especialização & Universidade & $20(33,3 \%)$ \\
& Periodontia & $20(33,3 \%)$ \\
& Implantodontia & $20(33,3 \%)$ \\
\hline${ }^{1}$ Forma de atualização na profissão & Cirurgia & $40(66,7 \%)$ \\
& & $30(50,0 \%)$ \\
& Congressos e jornadas & $30(50,0 \%)$ \\
\hline \multirow{2}{*}{ Realiza procedimentos cirúrgicos? } & Revistas científicas & $60(100,0 \%)$ \\
\hline \multirow{2}{*}{ Questiona durante a anamneses os medicamentos que estão } & Não & $9(15,0 \%)$ \\
\hline
\end{tabular}

Fonte: Autores.

Figura 1. Distribuição da amostra de acordo com a idade.

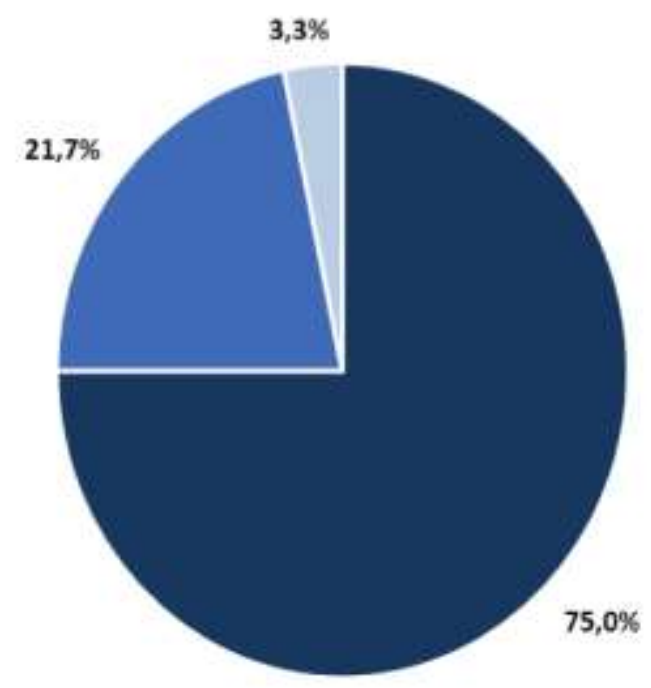

- Até 30 anos = De 31 a 49 anos = 50 anos

Fonte: Autores. 
Research, Society and Development, v. 10, n. 6, e20110615693, 2021

(CC BY 4.0) | ISSN 2525-3409 | DOI: http://dx.doi.org/10.33448/rsd-v10i6.15693

Figura 2. Distribuição da amostra de acordo com o local de atuação.

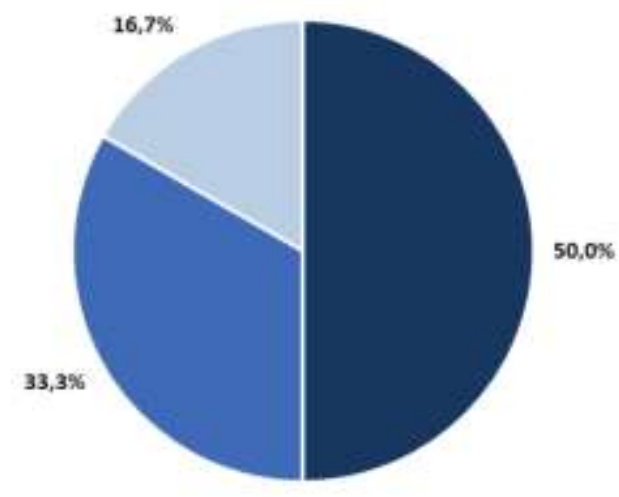

- Consultório particular = Serviço público de sáude = Universidade

Fonte: Autores.

Figura 3. Distribuição da amostra de acordo com a área de especialização.

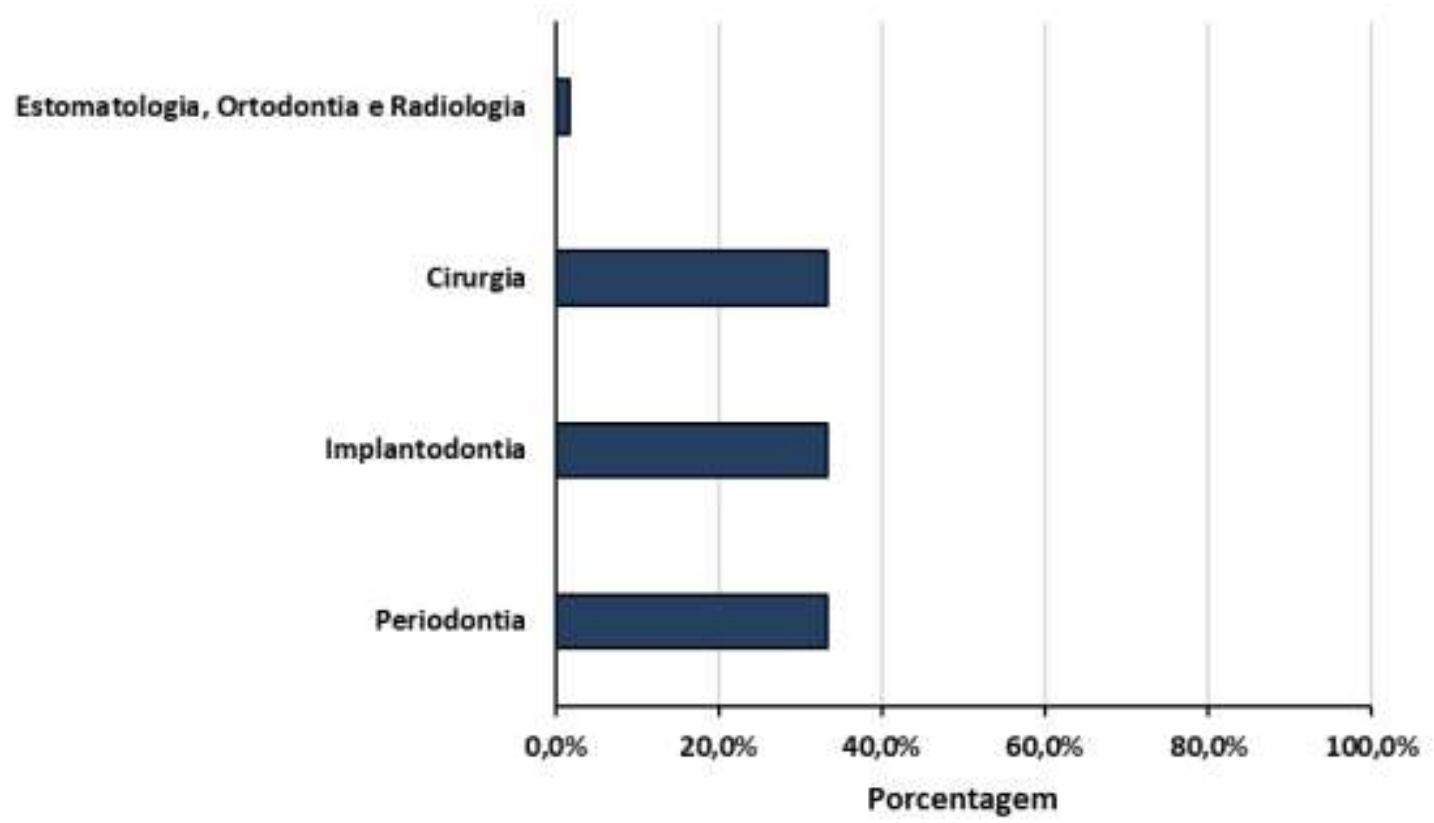

Fonte: Autores. 
Figura 4. Distribuição da amostra de acordo com a forma de atualização na profissão.

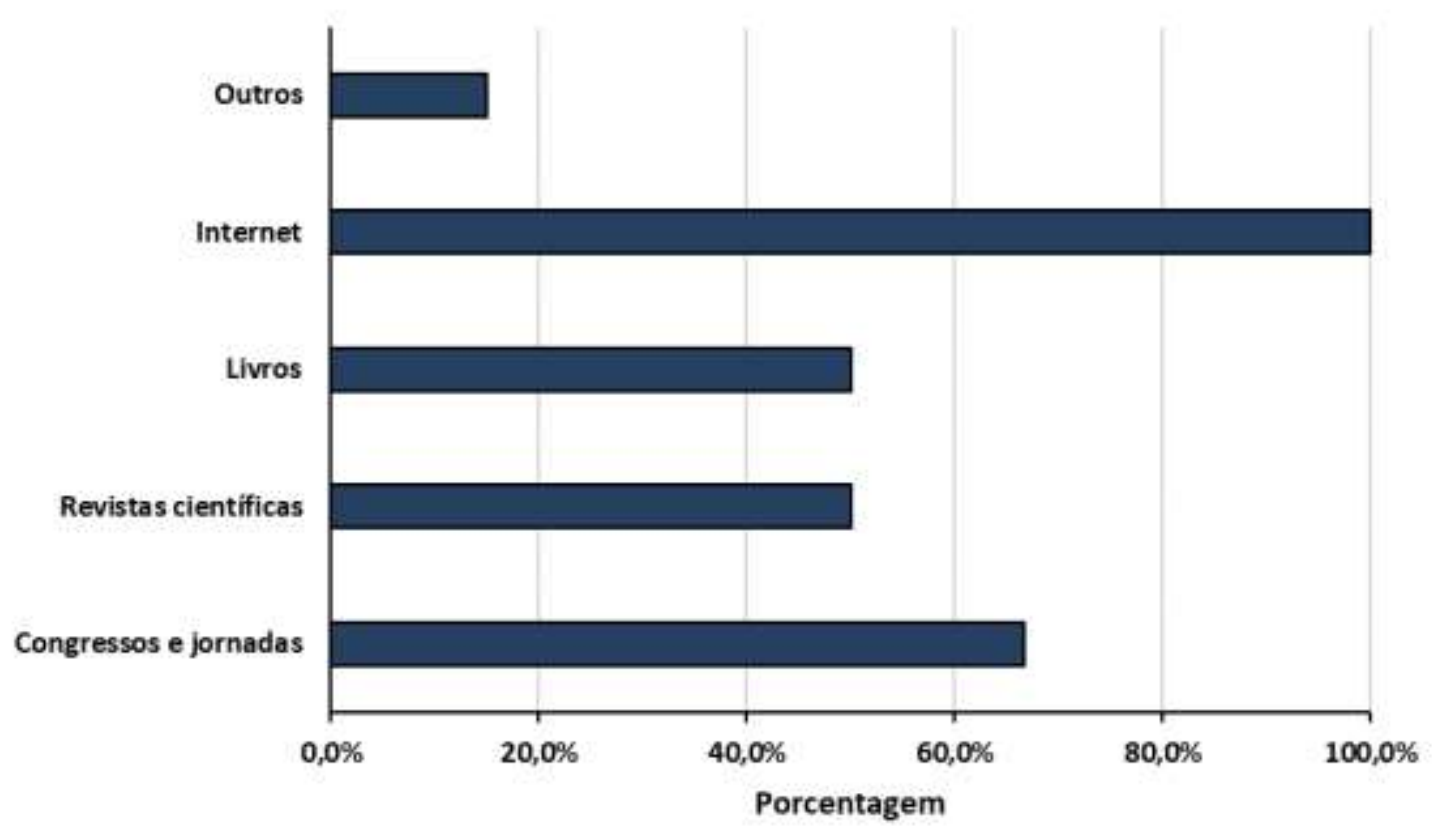

Fonte: Autores.

Na Tabela 2 e Figura 5 são apresentados o conhecimento da amostra acerca dos BFs e suas implicações.

Tabela 2. Análise descritiva das respostas sobre BFs.

\begin{tabular}{|l|l|c|}
\hline \multicolumn{1}{|c|}{ Variável } & \multicolumn{1}{|c|}{ Categorias } & \multicolumn{1}{c|}{$\begin{array}{c}\text { Frequência } \\
(\%)\end{array}$} \\
\hline Já atendeu pacientes que relatam o uso de BFs? & Não & $7(11,7 \%)$ \\
& Sim & $53(88,3 \%)$ \\
\hline \multirow{2}{*}{ Conhece ou já ouviu falar nas drogas do grupo dos } & Não & $4(6,7 \%)$ \\
BFs? & Sim & $50(83,3 \%)$ \\
& Não respondeu & $6(10,0 \%)$ \\
\hline \multirow{2}{*}{ Conhece as indicações dos BFs? } & Não & $20(33,3 \%)$ \\
& Sim & $30(50,0 \%)$ \\
Conhece as complicações orais implicadas no uso dos & Não respondeu & $10(16,7 \%)$ \\
BFs? & Não & $40(66,7 \%)$ \\
& Sim & $20(33,3 \%)$ \\
\hline
\end{tabular}

Fonte: Autores. 
Figura 5. Porcentagens de profissionais de acordo com as respostas sobre BFs.

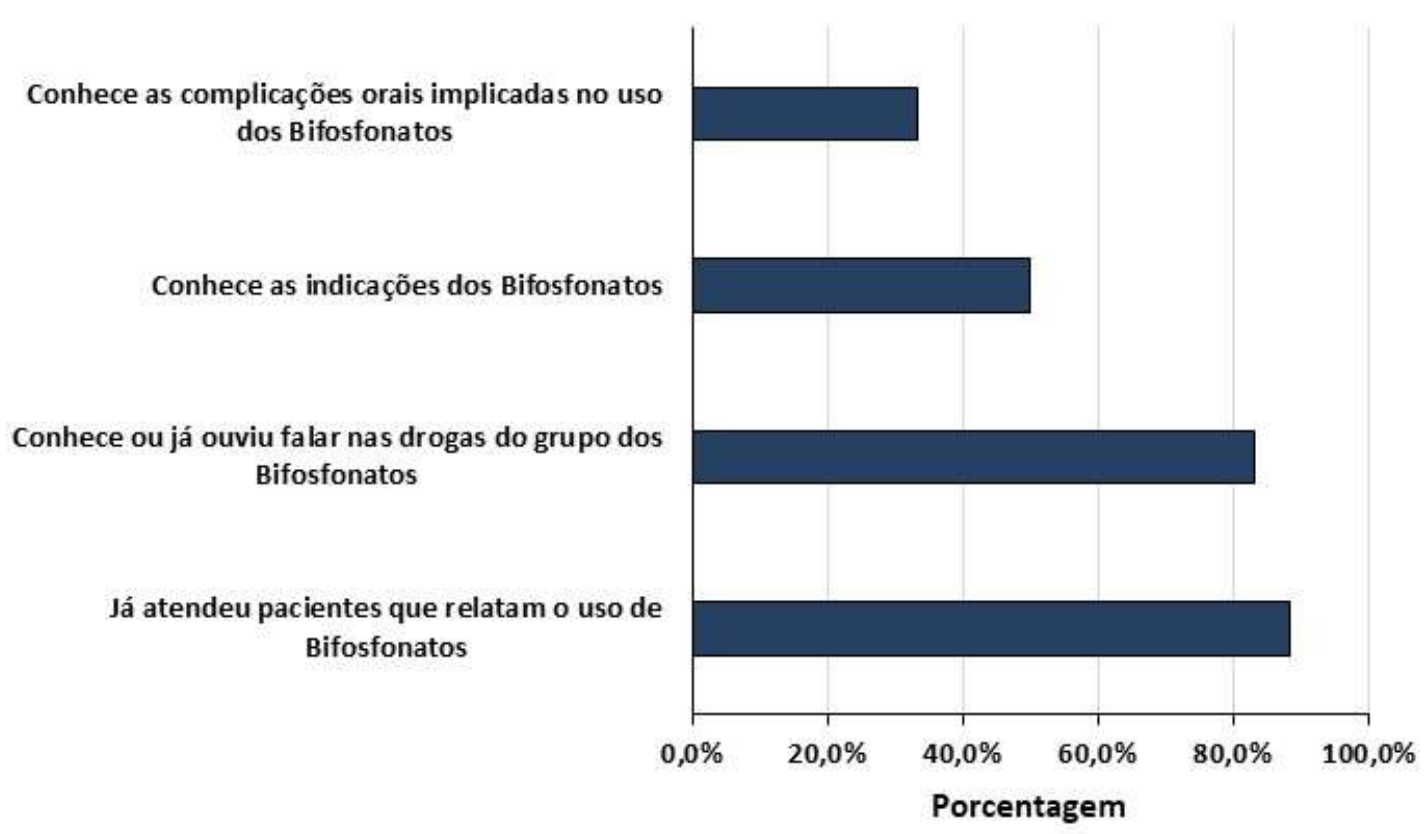

Fonte: Autores.

Observa-se que a maioria da amostra tem até 30 anos (75\%), com até um ano de formado (86,7\%). Nota-se ainda que 50,0\% atuam em consultórios particulares, 33,3\% em serviço público e 16,7\% em Universidades. As áreas de especialização dos profissionais da amostra são Periodontia, Implantodontia e Cirurgia. Observa-se que 66,7\% dos profissionais frequentam Congressos e Jornadas para sua atualização, 50,0\% em revistas científicas, 50,0\% livros e todos utilizam internet para atualização. Todos os profissionais (100\%) responderam que realizam procedimentos cirúrgicos, como por exemplo, exodontias ou cirurgias periodontais e que perguntam na anamnese de todos os pacientes quais os medicamentos que estes estão utilizando, antes de realizar qualquer procedimento.

Em relação aos BFs, 88,3\% dos profissionais já atenderam pacientes que relatam o seu uso e 83,3\% conhecem ou já ouviram falar. Ainda, 50\% respondeu que conhecem as indicações e somente 33,3\% desses conhecem as complicações orais do seu uso. Segundo Jornet (2010), 33.33\% da sua amostra souberam responder corretamente sobre como tratar a osteonecrose uma vez estabelecida, semelhante ao presente estudo onde 33,3\% dos Dentistas responderam como tratar ou conduzir a osteonecrose uma vez estabelecida. Quando questionados quais os tratamentos empregados em caso de complicações orais foram citados antibioticoterapia, câmera hiperbárica, cirurgia (tratamento cirúrgico radical - ressecção marginal e segmental) e uso de PDT (terapia foto dinâmica) com laser de baixa potência corado com azul de metileno para controle microbiológico e cicatrização tecidual.

Desta maneira, é importante salientar que mesmo a amostra avaliada do presente estudo possuindo especialização em áreas de procedimentos invasivos como Cirurgia, Implantodontia e Periodontia, uma minoria (colocar a porcentagem dessa minoria) soube responder as diversas formas de tratamento quando estabelecida a osteonecrose associada ao uso de BFs.

\section{Conclusão}

Com os resultados obtidos da presente pesquisa, ficou claro que mesmo havendo um número considerável de Cirurgiões Dentistas cientes da existência dos BFs e de seus possíveis efeitos adversos, em especial, a osteonecrose, poucos souberam quais tratamentos e condutas devem ser tomadas uma vez que a osteonecrose associada aos BFs está presente. 
Research, Society and Development, v. 10, n. 6, e20110615693, 2021

(CC BY 4.0) | ISSN 2525-3409 | DOI: http://dx.doi.org/10.33448/rsd-v10i6.15693

\section{Referências}

Brozoski, M. A. (2012). Osteonecrose maxilar associada ao uso de bisfosfonatos. Rev. Bras. Reumatol, 52(2): 265-270.

Albuquerque, T. P. Avaliação do grau de conhecimento dos Cirurgiões-Dentistas sobre osteonecrose dos maxilares associada ao uso de bisfosfonatos. 2014.46 p. Pesquisa Científica (Graduação em Odontologia) - Universidade Estadual da Paraíba.

Carvalho, A. (2008). Osteonecrose da mandíbula associada à bisfosfonatos intravenosos em doentes oncológicos. Acta Med Port, 21(5): 505-510.

Carvalho, L. N. V. (2018). Osteonecrose dos maxilares relacionada ao uso de medicações: Diagnóstico, tratamento e prevenção. Rev. CES Odont, 31(2): 4863.

Chianesi, A. C. M. importância dos bisfosfonatos na odontologia. 2018. 23 f. Trabalho de conclusão de curso (Graduação em Odontologia) - Centro Universitário São Lucas, Porto Velho, 2018.

Greenber, M. S. (2008). Intravenous bisphosphonates and osteonecrosis. Oral Surg Oral Med Oral Pathol Oral Radiol Endod, 98(3):259-260.

Hellstein, J. W. (2011). Managing the care of patients receiving antiresorptive therapy for prevention and treatment of osteoporosis: executive summary of recommendations from the American Dental Association Council on Scientific Affairs. J Am Dent Assoc, 142(11):1243-1251.

Hinchy, N. V. (2013). Osteonecrosis of the jaw - prevention and treatment strategies for oral health professionals. Oral Oncol, 49(9):878-886.

Kim, D. W. (2013). Osteonecrosis of the jaw related to everolimus: a case report. Br J Oral Maxillofac Surg, 51(8):e302-304.

Rigo, R. F. (2017). Osteonecrose perimplantarrelacionada ao uso de bisfosfonatos: Revisão de literatura. $R v A C B O, 26(1)$ : 31-37.

Rodan, G. A. (1996). Bisphosphonates: mechanisms of action. J Clin Invest, 97(12): 2692-2696.

Ruggiero, S. L. (2006). Practical guidelines for the prevention, diagnosis and treatment of osteonecrosis of the jaw in patients with cancer. J Clin Oncol Prac, 2(1): 7-14.

Sampaio, F. C. (2010). Mecanismos de Ação Dos Bisfosfonatos e sua Influência no Prognóstico do Tratamento Endodôntico. Rev. Fac. Odontol. 51(1): 31-38.

Tolentino, A. Avaliação do conhecimento dos cirurgiões-dentistas da rede pública do município de palhoça sobre bisfosfonatos e suas implicações no tratamento odontológico. 2018. 36 f. Trabalho de conclusão de curso (Graduação em Odontologia) - Universidade do Sul de Santa Catarina.

Yoo, J. Y. (2010). Survey of Korean dentists on the awareness on bisphosphonate-related osteonecrosis of the jaws. J. Investig. Clin. Dent. 1(9): 90-95. 